$\Lambda I A$ A $-97-4409$

\title{
ASCENT, TRANSITION, ENTRY, AND ABORT GUIDANCE ALGORITHM DESIGN FOR THE X-33 VEHICLE
}

\author{
John M. Hanson ${ }^{* \ddagger}$, Dan J. Coughlin ${ }^{\dagger \ddagger}$, Gregory A. Dukeman ${ }^{\dagger \ddagger}$, \\ John A. Mulqueen ${ }^{\ddagger}$, and James W. McCarter ${ }^{\ddagger}$ \\ NASA Marshall Space Flight Center \\ Huntsville, AL 35812

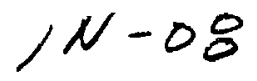 \\ 375989
}

Abstract

One of the primary requirements for X-33 is that it be capable of flying autonomously. That is, onboard computers must be capable of commanding the entire flight from launch to landing, including cases where a single engine failure abort occurs. Guidance algorithms meeting these requirements have been tested in simulation and have been coded into prototype flight software. These algorithms must be sufficiently robust to account for vehicle and environmental dispersions, and must issue commands that result in the vehicle operating within all constraints. Continual tests of these algorithms (and modifications as necessary) will occur over the next year as the X-33 nears its first flight. This paper describes the algorithms in use for X-33 ascent, transition, and entry flight, as well as for the powered phase of PowerPack-out (PPO) aborts (equivalent in thrust impact to losing an engine). All following discussion refers to these phases of flight when discussing guidance. The paper includes some trajectory results and results of dispersion analysis

\section{Introduction}

In June of 1996, Lockheed Martin SkunkWorks was selected for the Phase II contract of the design and development of the X-33 vehicle. Marshall Space Flight Center's Flight Mechanics, Guidance, Navigation and Control Systems Branch was selected by LMSW to develop the ascent, transition, entry and abort guidance algorithms for the X-33. These algorithms have been developed and documented and are undergoing simulation testing. Pending large changes in the vehicle design or subsystem behavior, the guidance algorithms architecture is complete.

The development of the guidance algorithms was challenging as they were being designed, coded, and tested in concurrence with the vehicle design and landing site selection phases. The ambitious schedule of the $\mathrm{X}$ 33 project demanded the co-development of on-board software during the hardware design phase. Guidance algorithms were required to handle all of the conceived mission profiles and single-engine failure abort missions. The $\mathrm{X}-33$ is a sub-orbital, single-stage, autoncmous, re-usable launch vehicle. It's purpose is to demonstrate the technologies necessary to develop the Reusable Launch Vehicle (RLV), eventually replacing the Space Shuttle.

The X-33 has the capability to launch vertically, achieve Mach 11, shutdown it's LOX-LH2 powered linear aerospike engines, and coast to a horizontal landing. The X-33 is scheduled to launch from Edwards Air Force Base (EAFB) in July, 1999. The primary landing site is at Michael Army Air Field (MAAF) in

\footnotetext{
- Chief, Flight Mechanics, Guidance, Navigation and Control Systems Branch, NASA Marshall Space Flight Center

${ }^{\dagger}$ Member, AIAA

' Aerospace Engineer, NASA Marshall Space Flight Center

Copyright $\odot 1998$ by the American Institute of Aeronautics and Astronautics, Inc. No copyright is asserted in the United States under Title 17. U.S. Code. The U.S. Government has royalty-free license to exercise all rights under the copyright claimed herein for Governmental Purposes. All other rights are reserved by the copyright owner.
} 
Ulah. approximately $360 \mathrm{nmi}$ dewnrange of the launch site.

There are a series of test thights (1) MAAF, each with specific govals to test the aerothermal protection system. To properly guide the vehicle through all of the mission profiles, the guidance and control system was developed as generically as possible. Through the use of premission I-loads, the vehicle has the capability to fly the numerous mission profiles and single-engine failure aborts corresponding to each of these nominal missions. This paper will provide an overview of the ascent, transition, and entry guidance algorithms for the X-33. Also to be discussed is the use of the Performance Monitor software for the single-engine abort scenarios.

\section{Ascent Guidance}

The guidance and control software resides on the Flight Manager (FM) processor on the vehicle. Ascent guidance is executed at $1 \mathrm{~Hz}$. Fig. 1 is a top-level flow diagram of the ascent guidance algorithm.

The Ascent_Guide function accepts the navigation state data and computes the expected relative velocity at the end of the guidance cycle using the current inertial velocity and acceleration vectors. The openloop function is executed from launch to a pre-determined time in the trajectory (tgr). The openloop function performs a table lookup of the steering attitude commands, throttle, and mixture ratio as a function of the computed relative velocity. In this manner, the guidance is always leading the control system.

The openloop function is followed by the acceleration tracking function. This function modifies the throttle command from openloop to track a nominal acceleration profile. This function reduces the Main Engine Cut-off (MECO) dispersions from uncertainties in engine performance and vehicle aerodynamics.

If the mission warrants the use of closed-loop ascent guidance, the function, IGM (Iterative Guidance Mode) is executed. IGM is the linear tangent steering law is the same as that used for the Saturn V (Iterative Guidance Mode--IGM, Reference 2). IGM has been slightly modified to accept relative targeting data necessary for the sub-orbital X-33 flights. The subfunction, igmguid, accepts targeting data computed in IGM and performs the attitude command calculations.

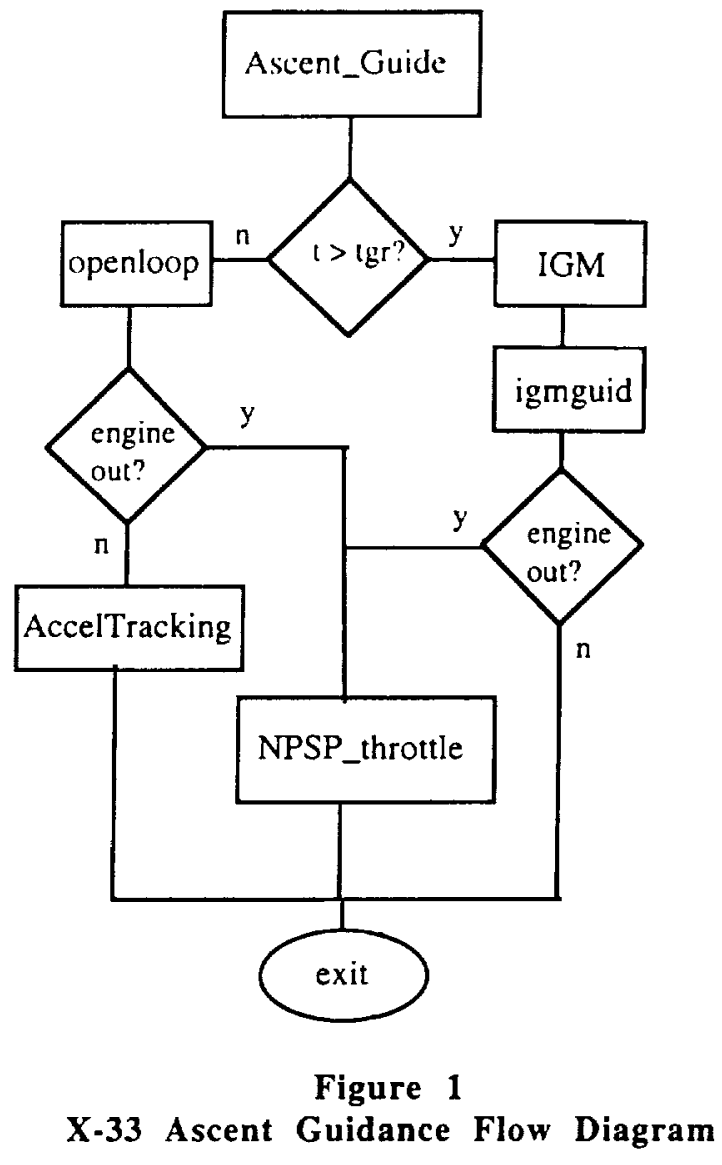

Unfortunately, the X-33 flights to MAAF do not have sufficient time in near-vacuum flight to warrant the use of IGM. Whether or not a closed-loop vacuum scheme is actually used on X-33, it will be needed for the follow-on VentureStar. ' IGM, while available for use, is not currently in use on any of the X-33 flight simulations. The primary reason for this is that the Performance Monitor provides a closed-loop procedure that is better for X-33 flight than IGM. This will be discussed later in this paper.

If an engine has failed during flight, the ascent guidance is responsible for computing the throttle level necessary to avoid cavitating the LOX pump. The LOX pump must maintain a minimum axial acceleration level (NPSP - Net Positive Suction Pressure) to prevent cavitation. This required acceleration level decreases as the throttle level decreases. While the engine would like a low throttle level, guidance requires a high

\footnotetext{
${ }^{\prime}$ Other guidance and control procedures needed for VentureStar, such as for automated rendezvous and dock, are also being worked at MSFC, but are not part of the scope of the X-33 effort.
} 
throule level as one engine (thus one half of the expecied acceleration) has failed. The function, NPSP_throttle, computes the highest possible throttle level which satisfies the acceleration requirements of the engine.

Throughout the ascent portion of flight, the current attitude is computed in the Ascent_Guide function given the attitude quaternion from navigation. The ascent guidance functions employ a launch inertial plumbline coordinate system. The attitude command is computed internally in the plumbline system and converted to a unit attitude quaternion and sent to the control system. Given the current and commanded attitude, the ascent guidance function also computes the body attitude rates necessary to achieve the commanded attitude by the next execution of Ascent_Guide.

The throttle command is also sent to the ascent control system where it is broken down into four chamber pressure commands. Ascent flight control has engine differential throttling and aerosurface deflection capability to control the attitude of the vehicle.

The mixture ratio command from ascent guidance is sent to the Propellant Utilization function. This function monitors the propellant levels in each tank and modifies the mixture ratio command to reduce the propellant residuals at MECO.

The $X-33$ has unique engine shutdown requirements. Traditionally, the engine shutdown command is issued from ascent guidance when the target MECO condition is achieved. A flight performance reserve in the form of propellant is

loaded on-board to guarantee that the target will be met despite vehicle and environmental uncertainties. However, the X-33 has grown in weight to the point that the landing gear will not support much residual propellant. Thus, the engine begins it's own shutdown procedure based on LOX depletion sensors located in the LOX feed lines. Ascent guidance continues to be executed until the engine shuts itself down. This effects $\mathrm{MECO}$ state dispersions in that propellant loading uncertainties extend or reduce the burntime of the engines.

\section{Transition Guidance}

Fairly simple transition guidance algorithm is specified vesired entry interface attitude is alphaTransitionDeg, and phibkTransitionDeg representing desired angle of attack and velocity bank angle magnitude. The appropriate bank sign to use is computed at transition initiation and is a simple function of the MECO velocity heading and the desired velocity heading (refer to Notation section)

$$
\operatorname{sgn}\left(\sigma_{c m d}\right)=-\operatorname{sgn}\left(\psi-\psi_{d}\right)
$$

See Figure 2 for definitions of heading angles. The bank sign thus computed is used throughout transition and subsequently used to initialize entry guidance bank command sign. In order to provide smooth bank and angle of attack commands, the output $\sigma_{c m d}$ of the filter represented by the following equation is passed as bank command to the attitude control system

$$
\ddot{\sigma}_{c m d}+2 \varsigma \omega_{n} \dot{\sigma}_{c m d}+\omega_{n}^{2}\left(\sigma_{c m d}-\sigma_{e i}\right)=0
$$

and analogously for angle of attack

$$
\ddot{\alpha}_{c m d}+2 \varsigma \omega_{n} \dot{\alpha}_{c m d}+\omega_{n}^{2}\left(\alpha_{c m d}-\alpha_{e i}\right)=0
$$

The filter states $\alpha_{c m d}$ and $\sigma_{c m d}$ are initialized during the first pass through transition guidance by setting the commanded values equal to the current estimated bank and angle of attack provided by the navigation subsystem and zeroing the filter rates

$$
\begin{aligned}
& \alpha_{c m d}=\alpha_{n a v}, \\
& \sigma_{c m d}=\sigma_{n a v}, \quad \text { (initialization) } \\
& \dot{\alpha}_{c m d}=0, \dot{\sigma}_{c m d}=0
\end{aligned}
$$

Commanded bank and angle of attack rates are generated, not by using the filter rates, but by comparing commanded angles with estimated angles and using a damping factor ( $1 / 2$ here) to reduce overshoot.

$$
\begin{aligned}
& \dot{\alpha}_{c m d}=\left(\alpha_{c m d}-\alpha_{n a v}\right) / 2 \\
& \dot{\sigma}_{c m d}=\left(\sigma_{c m d}-\sigma_{n a v}\right) / 2
\end{aligned}
$$

These rates are limited before being sent to the attitude control system

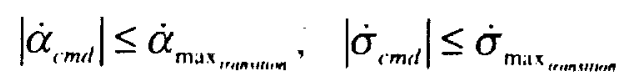




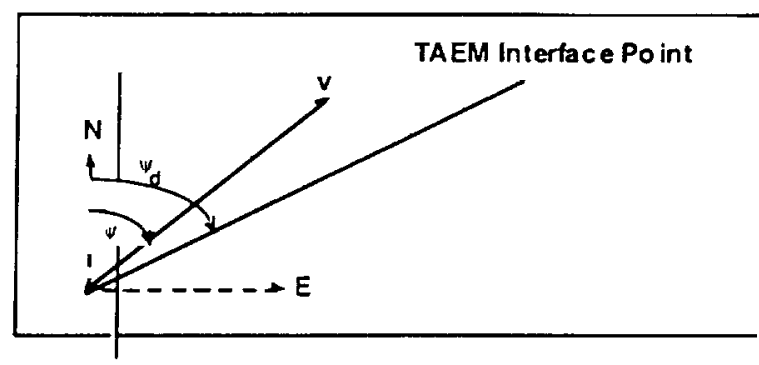

Figure 2

Heading Angles Definition_

\section{Entry Guidance}

The guidance algorithm controls range to a specified landing site by issuing bank angle commands which will cause the reentry vehicle to track a nominal drag acceleration versus relative energy profile. The drag profile is a by-product of the overall (ascent through entry) trajectory design process, using the Program to Optimize Simulated Trajectories (POST). All relevant constraints such as range, heating and dynamic pressure limits are enforced in the trajectory design process. This ensures that when the reentry vehicle flies the resulting nominal drag profile, all relevant entry constraints are satisfied. Lateral trajectory control is provided via bank reversal logic which is described later. Entry guidance is terminated at an Earth-relative velocity of $2,500 \mathrm{ft} / \mathrm{s}$.

This section is organized as follows. First, range (longitudinal) control equations and logic are developed. Then, synthesis of a nonlinear tracking control law is discussed in which bank angle modulation is used to follow a reference drag-energy profile. Next, heading (lateral) control equations are discussed.

Range Control The basic idea is to develop a nominal drag versus relative energy profile which can be tracked by the vehicle via bank angle modulation. For guidance purposes, the relevant equations of motion are those corresponding to planar motion about a non rotating Earth, with central gravity and aerodynamic lift and drag forces $^{3}$

$$
\begin{aligned}
& \dot{r}=v \sin \gamma \\
& \dot{v}=-D-g \sin \gamma \\
& v \dot{\gamma}=\left(v^{2} / r-g\right) \cos \gamma+D u
\end{aligned}
$$

where the control $\boldsymbol{u}$ is the vertical or in-plane component of lift to drag ratio
$u=(L / D) \cos \sigma$

The horizontal distance (range) corresponding to flight along a drag-energy profile can be computed as follows. Define relative energy

$e=v^{2} / 2+g h$

and the time rate of change of energy is

$$
\dot{e}=v \dot{v}+\dot{g} h+g \dot{h} \cong v(-D-g \sin \gamma)+g v \sin \gamma=-D v
$$

The time rate of change of range $R$ is given by

$$
\dot{R}=v \cos \gamma \cong v
$$

for near zero flight path angle (greater than about -5 degrees). Therefore, the predicted range for flight on the profile can be computed from:

$$
\frac{\partial R}{\partial e} \cong \frac{1}{D_{\text {ref }}} \Rightarrow R \cong \int_{e_{i}}^{e_{i}} \frac{1}{D_{\text {ref }}(e)} d e
$$

In practice, if the drag profile is stored and used as a piecewise linear function of relative energy, (12) is integrable and the range calculation is a simple summation involving logarithms of the stored reference drag values. The summation can be performed a priori to obtain nominal range-to-go values corresponding to the tabulated energy values., reducing the nominal range-to-go calculation each guidance cycle to a simple table lookup.

Note that the restriction on flight path angle, i.e., small magnitude, implies that the valid region of reentry for drag-energy guidance extends down to about Mach 2.5, where typically flight path angle starts becoming large negative. Figure 3 shows a typical drag-energy profile.

Tracking Control Law Synthesis In the following, the technique of feedback linearization is used to obtain an adaptive nonlinear tracking control law. Throughout the derivation of the control law, assume an exponential atmosphere model of the form

$$
\rho(h)=\rho_{0} \exp (-\beta h)
$$

and use the approximation that lime rate of change of drag coefficient is zero. (It is not difficult to remove the 
latter assumption and doing so does not appreciably complicate the control law.) Define tracking error, $z$, as the difference between (onboard) estimated drag acceleration and reference drag acceleration (from the stored drag profile):

$$
z(t)=D_{e s t}(t)-D_{r e f}(e)(14)
$$

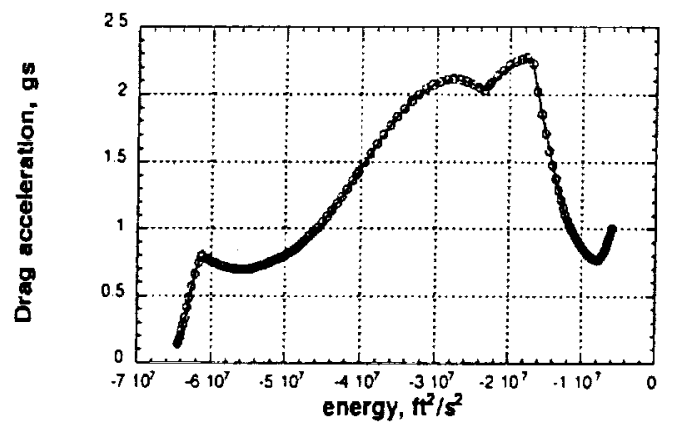

Figure 3

\section{Typical Drag-Energy Profile}

A desirable time response for the tracking error is the solution of the following second order differential equation (the well-known damped harmonic oscillator) with specified values of damping ratio and natural frequency:

$$
\ddot{z}(t)+2 \varsigma \omega_{n} \dot{z}(t)+\omega_{n}^{2} z(t)=0
$$

In the following, expressions are obtained for the first and second time derivatives of tracking error. It will be seen that the time derivative of flight path (gamma dot) shows up (linearly) in the second derivative from which an expression for "commanded" gamma dot is obtained. From commanded gamma dot, the corresponding gamma dot dynamical equation is used to obtain a commanded in-plane lift component. First, $z$ dot:

$$
\dot{z}(t)=\dot{D}_{e s t}-\dot{D}_{r e f}=-\beta D_{e s t} \dot{r}+\frac{2 D_{e s t}}{v} \dot{v}-D_{r e f}^{\prime} D_{e s t} v
$$

The primes denote differentiation with respect to relative energy. The second time derivative is:

$$
\begin{aligned}
& \ddot{z}(t)=-\beta D_{e s t}(\dot{v} \sin (\gamma)+v \cos (\gamma) \dot{\gamma})-\beta \dot{r} \dot{D}_{e s t}+ \\
& \frac{2 \dot{D}_{e s t}}{v} \dot{v}-2 \frac{D_{e s t}}{v^{2}} \dot{v}+2 \frac{D_{e s t}}{v}\left(-\dot{D}_{e s t r}-\dot{g} \sin (\gamma)-\right. \\
& g \cos (\gamma) \dot{\gamma}-D_{r e f}^{\prime}\left(\dot{D}_{e s t} v+D_{e s t r} \dot{v}\right) \\
& =-\beta\left(D_{e s t} \dot{v} \sin (\gamma)+\dot{r} \dot{D}_{e s t}\right)+\frac{2 \dot{v}}{v}\left(\dot{D}_{e s t}-\frac{D_{e s t}}{v}\right)- \\
& \frac{2 D_{e s t}}{v}\left(\dot{D}_{e s t}+\dot{g} \sin (\gamma)\right)- \\
& D_{r e f}^{\prime}\left(\dot{D}_{e s t} v+D_{e s t} \dot{v}\right)+\dot{\gamma}\left(-\beta D_{e s t} v \cos (\gamma)-2 \frac{D_{e s t}}{v} g \cos (\gamma)\right)
\end{aligned}
$$

It is assumed that the drag-energy curve will be in the form of a linearly-interpolated table (i.e., $D_{\text {ref }}^{\prime \prime}=0$ ). Now the harmonic oscillator equation can be formed:

$$
\begin{aligned}
& -\beta\left(D_{e s t} \dot{v} \sin (\gamma)+\dot{r} \dot{D}_{e s t}\right)+\frac{2 \dot{v}}{v}\left(\dot{D}_{e s t}-\frac{D_{e s t}}{v}\right)- \\
& \frac{2 D_{e s t}}{v}\left(\dot{D}_{e s t}+\dot{g} \sin (\gamma)\right)-D_{r e f}^{\prime}\left(\dot{D}_{e s t} v+D_{e s t} \dot{v}\right)+\dot{\gamma}\left(-\beta D_{e s t} v \cos (\gamma\right. \\
& \left.-2 \frac{D_{e s t}}{v} g \cos (\gamma)\right)+2 \zeta \omega\left(-\beta D_{e s t} \dot{r}+\frac{2 D_{e s t}}{v} \dot{v}-D_{r e f}^{\prime} D_{e s t} v\right)+ \\
& \omega_{n}^{2}\left(D_{e s t}-D_{r e f}\right)=0
\end{aligned}
$$

from which "commanded" gamma-dot is obtained:

$$
\begin{aligned}
& \dot{\gamma}_{c m d}= \\
& \frac{\left[\begin{array}{l}
\beta\left(D_{e s t} \dot{v} \sin (\gamma)+\dot{D}_{e s t}\right)+\frac{2 \dot{v}}{v}\left(-\dot{D}_{e s t}+\frac{D_{e s t}}{v}\right) \\
+\frac{2 D_{e s t}}{v}\left(\dot{D}_{e s t}+\dot{g} \sin (\gamma)\right)+D_{r e f}^{\prime}\left(\dot{D}_{e s t} v+D_{e s t} \dot{v}\right)- \\
2 \zeta \omega\left(-\beta D_{e s t} \dot{r}+\frac{2 D_{e s t}}{v} \dot{v}-D_{r e f}^{\prime} D_{e s t} v\right)-\omega_{n}^{2}\left(D_{e s t}-D_{r e f}\right)
\end{array}\right]}{-\beta D_{e s t} v \cos (\gamma)-2 \frac{D_{e s t}}{v} g \cos (\gamma)}
\end{aligned}
$$

Now this gamma-dot equation is equated with the gamma-dot equation (7) and solved for $u$, the inplane component of L/D:

$$
u=\left(v \dot{\gamma}_{r m d^{\prime}}-\left(v^{2} / r-g\right) \cos (\gamma)\right) / D_{e s t}
$$


The control law depends upon the current state $(r, v$, gamma), the drag-energy profile, the inverse scale height, beta, the estimated current drag acceleration and the design parameters omega and damping ratio, zeta. Note that the control law is independent of the current density and the current lift and drag coefficients. Thus, the control law has the interesting feature of not requiring a density model or an aerodynamic coefficient model. The estimated drag is just the component of sensed acceleration along the current navigated relative velocity vector. The sensed acceleration is equal to the accumulated velocity counts from the navigation system over the last one second.

To compensate for downrange dispersions, two steps must be taken. First of all, the Terminal Area Energy Management (TAEM) target is effectively biased such that the current range error that exists is increased by 50 percent. This is only used for control law evaluation purposes. This has the effect of driving the vehicle trajectory back to the nominal trajectory profile and preventing deviations from the nominal profile from growing as entry progresses. Secondly, the entire drag profile is (effectively) multiplied by the ratio of profile range-to-go to required range-to-go via:

$$
\begin{aligned}
& D_{\text {ref }}=\frac{R_{\text {nom }}(e)}{R_{\text {req }}} D_{\text {ref } f_{\text {nominat }}}(e) \\
& D_{\text {ref }}^{\prime}=\frac{R_{\text {nom }}(e)}{R_{\text {req }}} D_{\text {ref nominal }^{\prime}}^{\prime}(e)
\end{aligned}
$$

where $R_{\text {nom }}$ is the nominal range-to-go computed using the nominal drag profile. In the presence of heading error, a reasonable approximation of required range-to-go is given by (for a circle on a flat earth ${ }^{4}$ ):

$$
R_{r e q}=\frac{s \Psi_{e}}{\sin \left(\Psi_{e}\right)}
$$

where $\Psi_{e}$ is the current heading error The cyclically calculated values from (21) are the values of reference drag and slope that are used in the control law.

A value of 0.7 is highly recommended for the damping ratio ${ }^{4.5 .67 .8}$ In Ref. 6 the period (inversely proportional to $\omega_{n}$ ) is a function of drag acceleration level. A high value of drag implies that more aerodynamic force is available, which means that a faster response (smaller period) is possible for a given amount of bank maneuvering. In X-33 guidance, the period is a linear function of lift acceleration since it is the lift vector that is, in effect, being modulated (o) provide drag profile tracking.

In addition to the basic control law above (a proportional-derivative control law), an integral control increment is computed

$$
\Delta u=K \int_{t_{0}}^{t}\left(D_{e s t}-D_{r e f}\right) d t
$$

where the integration does not start until the transient dynamics have approximately died out, e.g. one period elapsed since closed-loop guidance initiation. The intent of the integral portion of the control law is to eliminate steady state error. ${ }^{5.6,7,8,9}$ The gain $\mathrm{K}$ on the integral term should be chosen very small to preserve the desired transient response properties as specified with the damping ratio and natural frequency. For the nonlinear system under consideration, engineering judgment and high-fidelity simulation testing are required to ensure that the integral portion of the control law enhances tracking performance and stability rather than degrades it.

Angle of attack is nominally commanded according to a pre-specified Mach-alpha schedule. Figure 4 shows a typical angle of attack profile. To augment bank angle control, angle of attack modulation about the nominal schedule is used. Assuming drag coefficient is linear with alpha, an incremental alpha, $\Delta \alpha$, required to make current drag equal to desired drag is

$$
\begin{aligned}
& D_{\text {ref }}-D_{e s t}=\Delta D= \\
& \frac{\rho v^{2} S \Delta C_{D}}{2 m}=\frac{\rho v^{2} S C_{D_{\alpha}} \Delta \alpha}{2 m}=\frac{D_{e s t} C_{D_{\alpha}} \Delta \alpha}{C_{D}} \\
& \Rightarrow \Delta \alpha=\frac{\Delta D C_{D}}{D_{e s t} C_{D_{u}}}
\end{aligned}
$$

This value is passed through a limiter to keep commanded angle of attack within five degrees of the nominal schedule. This alpha modulation capability is particularly useful during roll reversals and during shortperiod transients. An adverse effect of alpha modulation is that it's effect can sometimes be the opposite of what is desired. For example, to dectease drag, a lower value of alpha is commanded which also reduces lift which reduces altitude which increases drag. This adverse effect is especially noticeable when bank angle is saturated at all lift up. 
A bank angle increment, the intent of which is to drive the angle of altack profile back to the nominal schedule, is added to bank command":

$$
\phi_{r m d}=\cos ^{-1}((u+\Delta u) D / L)+4 \Delta \alpha
$$

The bank command is limited to between 0 and 85 degrees so that when a roll reversal is commanded, smaller maneuvers are required. The sign of the bank command is determined from the logic of the next section. Finally, to ensure that bank and angle of attack commands are reasonably consistent with vehicle attitude maneuver limitations, these two quantities are passed through a function that models the bank and angle of attack dynamics as second order systems with pre-specified rate and acceleration limits. This is analogous to the Transition Guidance logic. As in transition guidance, outputs to the control system are again bank, bank rate, alpha, and alpha rate.

Heading Angle Control The lateral logic is, for the most part, uncoupled from the longitudinal (range) control. During reentry, the lateral logic determines the sign of the commanded bank angle and does it independently of the range control (which determines the appropriate magnitude of the bank angle command).

In Ref. 6, a heading error versus speed corridor is stored onboard to determine when to command roll reversals. To minimize the extent to which the heading error corridor is tied to a specific entry profile, a new, more general approach has been developed for X33 reentry lateral control, a description of which follows.

The time rate of change of velocity heading angle is written ${ }^{3}$

$$
\begin{aligned}
& \dot{\psi}=-\frac{L \sin (\sigma)}{v \cos (\gamma)}+\frac{v}{r} \cos (\gamma) \sin (\psi) \tan (\phi)- \\
& 2 \omega(\tan (\gamma) \cos (\psi) \cos (\phi)-\sin (\phi))
\end{aligned}
$$

Likewise, an expression for the time rate of change of desired heading (omitted here for brevity) can be obtained from its definition so that the time rate of change of heading error is

$$
\begin{aligned}
& \dot{\psi}_{c}=\dot{\psi}_{d}-\dot{\psi}=\dot{\psi}_{d}-\frac{L \sin (\sigma)}{v \cos (\gamma)}+ \\
& \frac{v}{r} \cos (\gamma) \sin (\psi) \tan (\phi)- \\
& 2 \omega(\tan (\gamma) \cos (\psi) \cos (\phi)-\sin (\phi))
\end{aligned}
$$

Equating to zero, and solving for bank angle gives

$$
\begin{aligned}
& \sin (\sigma)=\dot{\psi}_{d}+\frac{\nu^{2}}{L r} \cos ^{2}(\gamma) \sin (\psi) \tan (\phi)- \\
& 2 \omega \frac{\nu \cos (\gamma)}{L}(\tan (\gamma) \cos (\psi) \cos (\phi)-\sin (\phi))
\end{aligned}
$$

This value is the bank angle required to maintain the current heading error. If the magnitude of the required bank is greater than 12 degrees, then a sign change (on the commanded bank angle) is indicated.

When Mach decreases to below 4.0, bank angle is no longer determined from range control considerations but rather from heading consideration, i.e., bank angle command is proportional to the current heading error.

$$
\sigma=4\left(\psi_{d}-\psi\right)
$$

\section{Performance Monitor}

On-board the X-33, a separate computer contains the Mission Manager (MM) logic. The Mission manager detects the current phase of flight and executes the onboard Performance Monitor (PM) which is a 3 degreeof-freedom X-33 simulation that predicts the trajectory from the current vehicle state to the TAEM interface. Under certain conditions, the PM may send commands to the FM which modifies the nominal guidance steering command to the control system. In these situations the MM/PM functions as a closed-loop guidance algorithm.

One of the duties of the Mission Manager is to detect the depletion of propellant and signal a change of flight mode fron the ascent to transition phase of flight. Another important role of the Mission Manager is to detect an engine out situation and, based on the time of failure, use the Performance Monitor to reshape the trajectory to the nominal landing site, reset the guidance targets to divert to an alternate landing, or predict the need for an intentional destruction of the vehicle. 
The Performance Monitor is a 3-DOF simulation that is intended $t o$ be llown on-board the X-33 vehicle as par of flight software. The Performance Monitor will be executed continuously, initializing to current flight state data and executing until the TAEM target conditions are either missed or achieved. These results will provide a prediction, in real time, whether a safe landing at the nominal landing site can be achieved. The Performance Monitor will also be used to assess the reshaped trajectory for an open-loop ascent abort before allowing the guidance system to use the new real-time computed chi tables. The on-board trajectory reshaping problem is complicated by a minimum acceleration constraint on the engine to avoid pump cavitation. The minimum acceptable axial acceleration is a function of the remaining liquid oxygen and a function of the throttle level.

The MM uses a drag factor parameter as an indicatror of how much energy margin the vehicle will have at the beginning of the entry phase. The drag factor is calculated within the PM and is the ratio of the drag profile range to the required range to reach the nominal TAEM conditions. The drag profile range is the downrange distance that the vehicle could achieve if at the current energy condition, the vehicle followed the nominal drag profile. The required range is the great circle range from the current vehicle position to the TAEM point. The drag factor decreases significantly for early engine pump failure times because the energy level at MECO becomes much lower.

Due to the decreased vehicle thrust to weight ratio if one of the two linear aerospike engines fails early in flight it is not always possible to reach the nominal or altemate landing site. In this case the Mission Manager would predict the need to destroy the vehicle. Fortunately, the vehicle spends a considerable amount of time over the EAFB site so the vehicle impact point would be well within EAFB boundaries. The minimum throttle range constraint does not allow for RTLS-type trajectories.

Figure 5 shows the final range to $\mathrm{HAC}$ as a function of drag factor. It was found that when the drag factor is above 0.7 , the closed loop entry guidance could successfully achieve TAEM conditions without any reshaping of the ascent trajectory by the Performance Monitor. This corresponds to single engine failure times greater than 40 seconds and all of the likely vehicle and trajectory dispersions that have been studied. lofted. This lofiting is induced beciause at a given altitude, the vehicle has less velocity than it would on a nominal trajectory. Since the ascent open loop attitude commands (Chi Table) are based on velocity, the pitch commands at a given altitude are higher than in the nominal case. For late engine failure times entry guidance can compensate for the lower energy state at MECO. However, for early failure there is not suffucient energy to achieve the TAEM targets unless the ascent trajectory is modified.

The trajectory profiles for early engine failures show the effect of Performance Monitor reshaping on the ascent trajectories. Initially, just after the pump failure, the Performance Monitor reshaping logic reduced the value of the pitch command from the open loop Chi table. This reduces the trajctory lofting. During the later portion of the ascent trajectory the value of the pitch command is increased. This modification (or reshaping) of the pitch commands in the nominal Chi table enables the vehicle to reach an acceptable energy level at MECO which allows entry guidance to achieve the target TAEM conditions.

The use of an on-board 3-DOF vehicle simulation to evaluate, in real time, the ability of the guidance system to achieve its intended target objective has not been attempted before on any launch vehicle or spacecraft. This innovative X-33 flight software design will add significant "intelligence" to the GN\&C system.

\section{Dispersion Results}

The ascent, transition and entry guidance algorithms were tested in a 3 DOF simulation against environmental and system dispersions. The various parameters were dispersed in a Monte Carlo fashion. Dispersion studies were ran for three of the five test flights to MAAF. The dispersion results to be presented here are from the third flight: Michael $7 \mathrm{c} 3$. This purpose of this particular flight is to reach a specific level of catalytic aeroheating. Table 1 lists the parameters which were dispersed and the level of their dispersions. An EAFB 3 hr wind-pair database was used for this dispersion analysis. A wind-biased trajectory was designed for the first of the wind and simulated against the second. The GRAM 97 (Global Reference Atmosphere Model) was employed to randomly vary the atmosphere for a given month and the winds during entry. Results here will be limited to the set of January simulations.

The trajectory profiles in Figure 6 indicate that the trajectories for failure times greater than 40 seconds are 


\begin{tabular}{|l|c|}
\hline $\begin{array}{l}\text { Dispersion } \\
\text { Paramelers }\end{array}$ & $\begin{array}{c}\text { Dispersion } \\
\text { Level }\end{array}$ \\
\hline Aerospike Engine & \\
\hline $\begin{array}{l}\text { Mixture Ratio Error } \\
\text { (LOX:LH2) }\end{array}$ & $\pm 2 \%$ \\
\hline Fx Engine Thrust Error & $\pm 1 \%$ \\
\hline LOX Flow Rate Error & $\pm 0.6 \%$ \\
\hline LH2 Flow Rate Error & $\pm 1.125 \%$ \\
\hline Environments & \\
\hline Ascent Atmosphere & $\begin{array}{c}\text { GRAM 97 EAFB } \\
\text { Jan, July \& Nov }\end{array}$ \\
\hline Entry Atmosphere & $\begin{array}{c}\text { GRAM 97 } \\
\text { Jan, July \& Nov }\end{array}$ \\
\hline Ascent Winds & $\begin{array}{c}2 \text { nd of } \\
\text { EAFB DOL }\end{array}$ \\
\hline Entry Winds & $\begin{array}{c}\text { Randomly } \\
\text { Dispersed Wind } \\
\text { Profile }\end{array}$ \\
\hline Mass Properties & \\
\hline Reentry Mass Error & $\pm 0.6 \%$ \\
\hline Loaded LOX Error & $\pm 0.232 \%$ \\
\hline Loaded LH2 Error & $\pm 0.181 \%$ \\
\hline $\begin{array}{l}\text { Vehicle } \\
\text { Aerodynamics }\end{array}$ & $\begin{array}{c}\text { Table } 1 \\
\text { CA/CN }\end{array}$ \\
\hline $\begin{array}{l}\text { Navigation } \\
\text { Errors }\end{array}$ & $\begin{array}{c}\text { Navigation } \\
\text { uncertainty model } \\
\text { specified in } \\
\text { Document v1.1 }\end{array}$ \\
\hline Dispersion Parameters \\
\hline
\end{tabular}

Many output parameters were tracked during each of the Monte Carlo simulations. Ascent loading indicators, MECO parameters such as cutoff time, altitude, Mach number, and flight path angle. Entry parameters were also tracked including thermal and structural indicators. The results presented in this paper will be limited to the parameters recorded at the TAEM interface (defined when the relative velocity reaches $2500 \mathrm{ft} / \mathrm{s}$ ). In this manner, we can judge how well the ascent, transition and entry guidance steered the vehicle to the final target.. The tracked TAEM parameters are listed in table 2.

The guidance performed well under the given dispersion levels. There were extreme cases that required further investigation but the standard deviation values were with the TAEM target zone.

Figure 7 is a plot of the maximum and minimum crossrange as a function of the vehicle downrange for

this set of disperison simulations. Again, the groundtrack spread was within acceptable values.

\author{
Variable \\ Taem Time \\ altFT \\ Mach \\ fpaDeg \\ rangeLand
}

\section{Description}

Trajectory time at which the TAEM condition is reached (s) Altitude at TAEM ( $\mathrm{ft}$ ) Mach number at TAEM Flight path angle at TAEM (deg) range to the TAEM point (nmi)

Table 2

Tracked TAEM Dispersion Parameters

\begin{tabular}{|l|c|c|c|c|}
\hline & Min & Max & Average & Stdev \\
\hline $\begin{array}{l}\text { Taem } \\
\text { Time }\end{array}$ & 428 & 464 & 445.6 & 5.5 \\
\hline altFt & 84394.1 & 101439 & 94018.4 & 2044.6 \\
\hline Mach & 2.44 & 2.68 & 2.57 & 0.05 \\
\hline rangLand & 0.1 & 19.9 & 1.67 & 1.44 \\
\hline fpaDeg & -17.8 & -6.9 & -11.2 & 1.4 \\
\hline
\end{tabular}

Table 3

Michael 7c3 Trajectory Dispersion Results

\section{References}

1. Dukeman, G A., and Gallaher, M.W., "Guidance and Control Concepts for the X-33 Technology Demonstrator", paper AAS-98-026, 21st Annual AAS Guidance and Control Conference, Feb. 4-8, 1998, Breckenridge, $\mathrm{CO}$

2. Smith, I.E., "General Formulation of the Iterative Guidance Mode," NASA TM X-53414, March 22, 1966.

3. Vinh, Nguyen, Optimal Trajectories in Atmospheric Flight. Chapter 3, Elsevier Scientific Publishing Company, 1981.

4. Tannas, Lawrence E. Jr., and Perkins, Toney R., "Simulation Evaluation of Closed Form Reentry Guidance", AIAA Guidance, Control and Flight Dynamics Conference, 1967, AIAA Paper 67-597.

5. D'Azzo, John J., and Houpis, Constantine H., Linear Control System Analysis and Design. Chapters 10, 15, 2d ed., 1981. 
6. Harpold, Jon C., and Graves, Claude A. Jr., "Shuttle Entry Guidance", Journal of the Astronautical Sciences", Vol. XXVII, No. 3, pp. 239-268, JulySeptember, 1979.

7. Lu, Ping, "Entry Guidance and Trajectory Control for Reusable Launch Vehicle", Journal of Guidance, Control and Dynamics, Vol. 20, No. 1, JanuaryFebruary, 1997, pp. 143-149.
8. Mease, Kenneth M., and Kremer, Jean-Paul, "Shuttle Entry Guidance Revisited Using Nonlinear Geometric Methods", Journal of Guidance, Control, and Dynamics, Vol. 17, No. 6, 1994, pp. 1350-1356.

9. Doyle, John C., Francis, Bruce A., and Tannenbaum, Allen R., Eeedback Control Theory, Chapter 3, 1992.

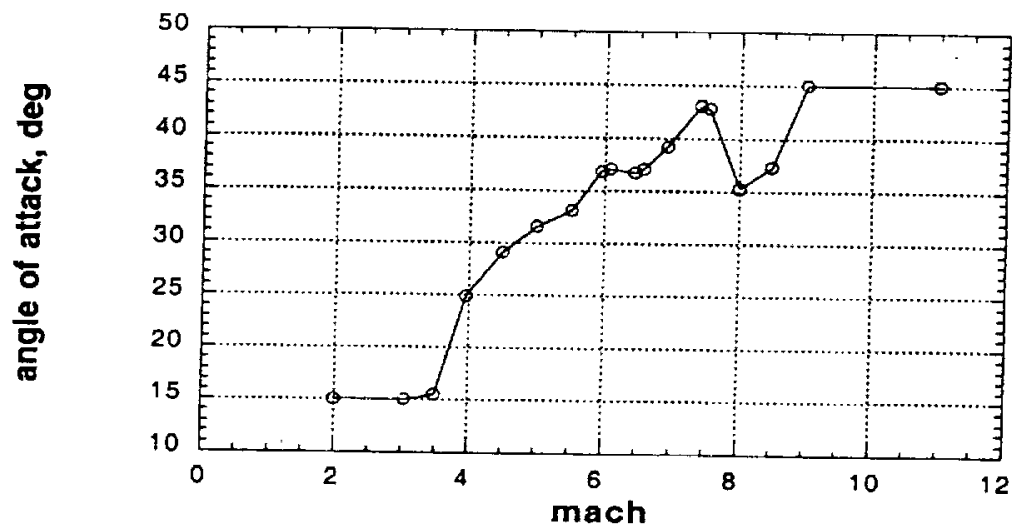

Figure 4

Typical Entry Angle of Attack Profile

Range to $H A C$ as a Function of Drag Factor for the Michael 7c7 X-33 Mission

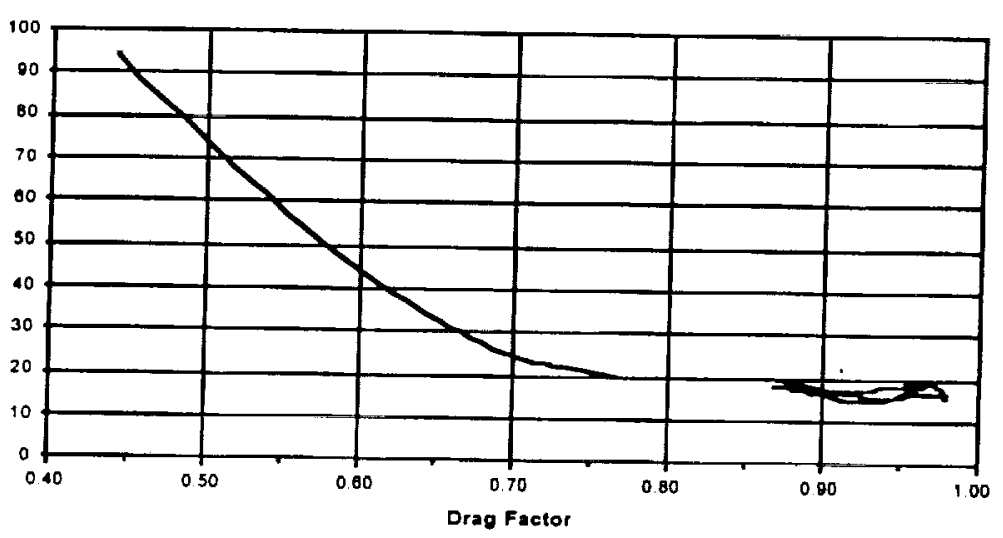

Figure 5

Range to HAC as a Function of Drag Factor 
X-33 Power Pack Failure Guided Abort Trajectories tc Michael

(With Performance Monitor Reshaping)

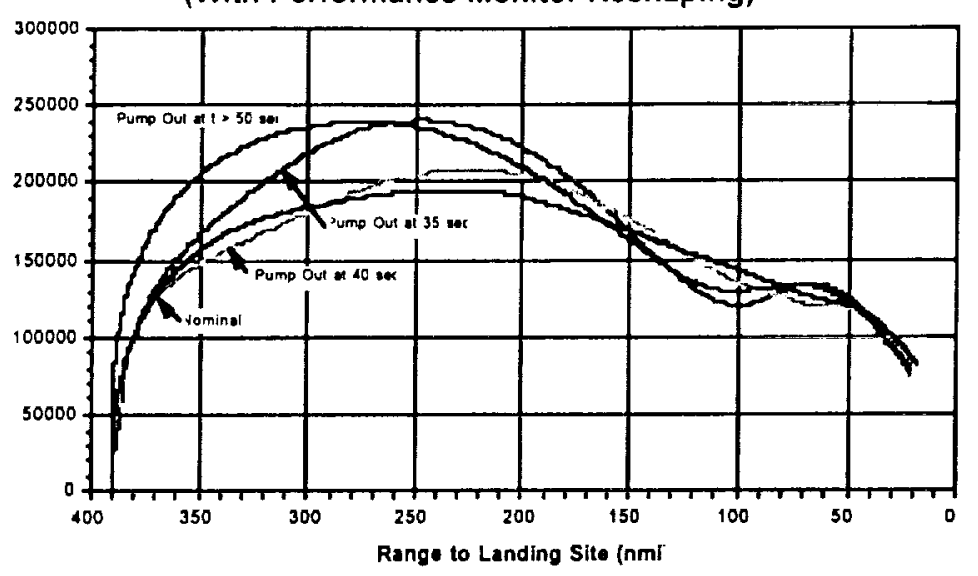

Figure 6

X-33 Nominal and Abort Trajectory Profiles

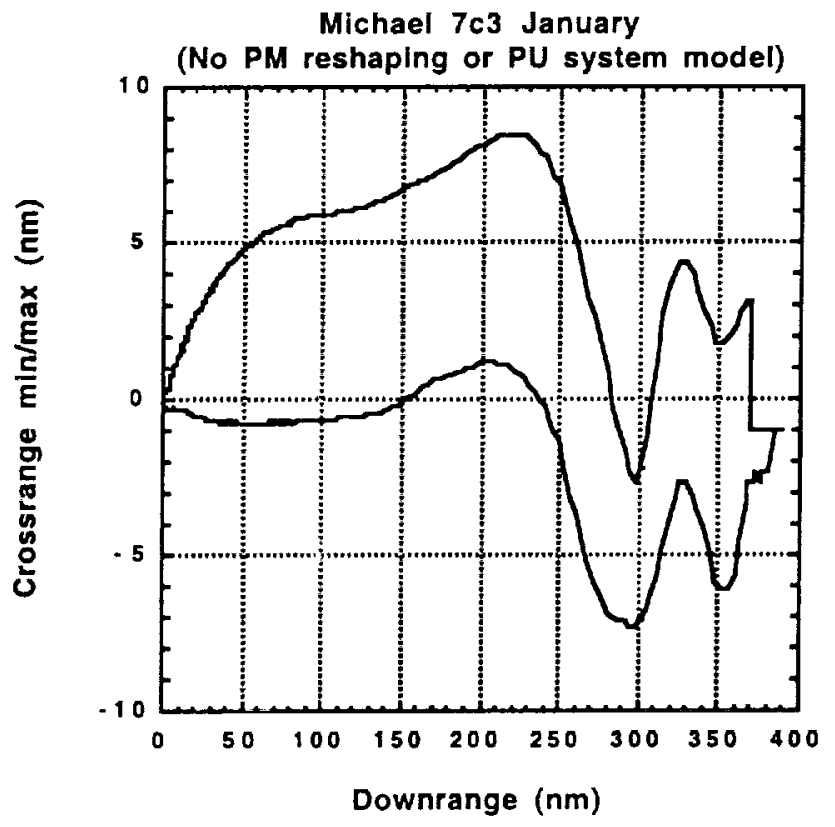

Figure 7

X-33 Trajectory Dispersion Results 